\title{
Disease and Diagnosis
}

\section{Clinical, Radiological, and Laboratory Findings in Patients Infected With 2019 Novel Coronavirus (SARS- CoV-2)}

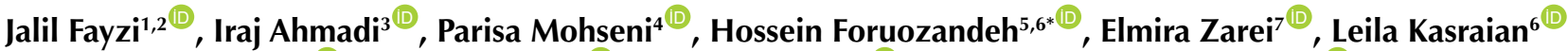 \\ , Zahra Foruozandeh ${ }^{8}{ }^{\circledR}$, Abbas Farahani $^{\oplus}{ }^{\oplus}$, Hossein Hafezi ${ }^{10}$, Mehdi Gholamzadeh Baeis ${ }^{11^{\circledR}}$, Mohammad \\ Rafi Bazrafshan $^{12}{ }^{\circledR}$, Sara Bakhtiari Nezhad ${ }^{13}{ }^{\circledR}$, Hamideh Mohseni ${ }^{10}$ \\ ${ }^{1}$ Infectious Diseases Specialist, Assistant Professor, Department of Infectious Diseases, School of Medicine, Ilam University \\ of Medical Sciences, Ilam, Iran. \\ ${ }^{2}$ Clinical Research Department, Mostafa Khomeini Hospital, Ilam University of Medical Sciences, Ilam, Iran. \\ ${ }^{3}$ Department of Physiology, School of Medicine, Ilam University of Medical Sciences. Ilam, Iran. \\ ${ }^{4}$ Student Research Committee, Department of Epidemiology, School of Public Health and Safety, Shahid Beheshti \\ University of Medical Sciences, Tehran, Iran. \\ ${ }^{5}$ Cellular and Molecular Research Center, Gerash University of Medical Sciences, Gerash, Iran. \\ ${ }^{6}$ Blood Transfusion Research Center, High Institute for Research and Education in Transfusion Medicine, Shiraz, Iran. \\ ${ }^{7}$ Tehran University of Medical Sciences (TUMS), Tehran, Iran. \\ ${ }^{8}$ Department of Nursing, Jahrom University of Medical Sciences, Jahrom, Iran. \\ ${ }^{9}$ Infectious and Tropical Diseases Research Center, Hormozgan Health Institute, Hormozgan University of Medical \\ Sciences, Bandar Abbas, Iran. \\ ${ }^{10}$ Department of Dermatology, Hormozgan University of Medical Sciences, Bandar Abbas, Iran. \\ ${ }^{11}$ Department of Radiology, Imam Hossein Hospital, Shahid Beheshti University of Medical Sciences, Tehran, Iran. \\ ${ }^{12}$ Department of Nursing, School of Nursing, Larestan University of Medical Sciences, Larestan, Iran. \\ ${ }^{13}$ Islamic Azad University of Tehran Shomal, Tehran, Iran. \\ ${ }^{14}$ Larestan Branch, Islamic Azad University, Larestan, Iran.
}

\begin{abstract}
The outbreak of a novel coronavirus disease 2019 (COVID-19) began in China in December 2019 and spread worldwide. The current review summarized clinical, radiological, and laboratory findings of patients infected with COVID-19. Based on many studies, the main symptoms of the disease include respiratory symptoms, fever, cough, and dyspnea; there is also a wide range of biochemical, hematological, and radiological changes in the patients. The signs (or symptoms) and other variables in the early stage or the mild stage of the disease appear in a highly heterogeneous and non-specific manner. Identifying the clinical and paraclinical symptoms of COVID-19 can be effective in controlling it.

Keywords: Coronavirus disease 2019, Severe acute respiratory syndrome, Clinical symptoms,

Laboratory findings, Radiological features
\end{abstract}

*Correspondence to Hossein Foruozandeh, Cellular and Molecular Research Center, Gerash University of Medical Sciences, Gerash, Iran. Blood Transfusion Research Center, High Institute for Research and Education in Transfusion Medicine, Shiraz, Iran. Tel: 00989382109998; Email: hosainforuoandeh@yahoo. com

Received: September 9, 2020, Accepted: December 6, 2020, ePublished: December 30, 2020

\section{Introduction}

The outbreak of coronavirus disease 2019 (COVID-19) began in Wuhan, China in December 2019, and eventually turned into a worldwide pandemic (1). It is caused by becoming infected with a specific type of coronavirus. The International Committee of Taxonomy of Viruses (ICTV) named this virus as severe acute respiratory syndrome coronavirus 2 (SARS-CoV-2) (2). Coronaviruses (CoVs) are a group of RNA viruses that cause diseases in mammals and birds. They are single-stranded positive-sense RNA viruses belonging to the Coronaviridae family (3).
Asymptomatic carriers of the coronavirus are the main source of the virus (4). COVID-19 spreads most often when an infected person is in close contact with another person. The virus spreads through the air, primarily via respiratory droplets or aerosols, when the infected person sneezes, coughs, speaks, or breathes heavily. Moreover, it may be transmitted via contaminated surfaces (4-6). The incubation period may range from 1 to 14 days but mostly occurs from day 3 to 7 (7). The disease is associated with clinical symptoms of viral pneumonia, and the most common symptoms are fever, fatigue, dry coughs,

(C) 2020 The Author(s). This is an open access article distributed under the terms of the Creative Commons Attribution License (http:// creativecommons.org/licenses/by/4.0/), which permits unrestricted use, distribution, and reproduction in any medium, provided the original work is properly cited. 
and shortness of breath $(6,8)$. Other symptoms, such as runny nose, nasal congestion, and diarrhea have been reported less frequently; in more severe cases, dyspnea is also seen. Moreover, acute respiratory distress syndrome (ARDS), septic shock, difficulty to compensate metabolic acidosis, and coagulative dysfunction develop rapidly (9). COVID-19 has a mild to severe spectrum. Also, mortality rates vary from country to country, and it has been reported between $0.4 \%-3.4 \%$ (10). On January 30, 2020, the World Health Organization (WHO) announced the epidemic of COVID-19 as a global health emergency (11).

Due to the lack of a completely effective drug or vaccine for COVID-19, timely identification of patients and their isolation from the healthy population can prevent its rapid spread (12). Currently, molecular tests are the standard methods to confirm COVID-19, but in negative reverse transcription-polymerase chain reaction (RT-PCR) test patients, a combination of travel history of the individual to the affected areas as well as clinical symptoms along with typical CT imaging and laboratory findings are used $(12,13)$. Moreover, using biosensors in detecting COVID-19 is a new view that can improve the diagnostics procedure in the future (14). The current review focused on the manifestations and symptoms of COVID-19 in various tests.

\section{Clinical Findings}

People with COVID-19 have had a wide range of symptoms. Fever (88\%-98\%), dry coughs (60\%-82\%), dyspnea (31\%-55\%), chest tightness (31\%-40\%), sudden loss of smell (anosmia) and/or taste (ageusia) (30\%-60\%) are the most common symptoms of the disease (15-18). Other signs and symptoms included: sputum production (28\%-29.33\%), fatigue (22\%-44\%), myalgia (11\%-44\%), headache $(8 \%)$, haemoptysis (5\%), diarrhea (2\%-3\%), poor appetite $(12.0 \%)$, shortness of breath, rash, sore throat, face pain, nasal obstruction, throat congestion, tonsil swelling, enlargement of lymph nodes, vomiting, consciousness disorder, paresthesia, and stomachache (3, 18-24). Severe cases of COVID-19 may experience worsened symptoms, such as coagulation dysfunction, irreversible bleeding, ARDS, septic shock, and refractory metabolic acidosis (21).

\section{Molecular Test}

Nucleic acid testing is the primary method of diagnosing COVID-19. It is done by RT-PCR or determining the viral gene sequence in the sputum, throat swabs, feces, or blood samples $(2,6)$.

Respiratory tract sampling is the best way to perform the COVID-19 diagnosis test. Until recently, the specimens were taken mostly from the lower respiratory tract (3). But, sampling of the lower respiratory tract is more difficult and requires skilled medical staff. It can also cause airway damage and bleeding and is painful for the patients. Instead, collecting upper respiratory specimens included nasopharyngeal, oropharyngeal, and nasal swab specimens is safe, rapid, and straightforward (20). Therefore, several researchers investigated the bio-distribution of COVID-19 in different samples and different stages after illness onset.

In a study conducted by Wang et al , the biodistribution of 2019-nCoV in different types of clinical specimens was investigated using real-time RT-PCR methods. Their findings showed that bronchoalveolar lavage fluid specimens (93\%), sputum (72\%), and nasal swabs (63\%) had the highest positive rates, respectively; and fiber bronchoscope brush biopsy (46\%), pharyngeal swabs (32\%), feces (29\%), and blood (1\%) followed them. Moreover, none of the urine samples were positive (21).

Yang et al showed that in the first 14 days after onset of the disease the sputum (74.4\%-88.9\%) and nasal swabs (53.6\%-73.3\%) had the highest positive rate, respectively. Sputum and nasal swabs had a positive rate ranging from $42.9 \%$ to $61.1 \%$, 15 days after disease onset. Moreover, the positive rate of throat swabs was low 8 days after disease onset (22).

Various studies have shown that the positive rate of RT-PCR for throat swab specimens was about 30$60 \%$. It depends on the procedure of sample collection, transportation, specimens' source, sampling time (different period after disease onset), and the type of utilized kit (20-23).

Zhang et al evaluated the patients during the medical treatment period using molecular methods. Their finding indicated that $53.3 \%$ had positive oral swabs, $26.7 \%$ had positive anal swabs, $40 \%$ were blood positive, $20 \%$ were serum positive, and 2 patients had both anal and oral swabs positive on day 0 (25). On day 5 , they found only $25 \%$ positive oral swabs. In contrast, $37.5 \%$ of anal swabs were positive. These findings suggested that at the beginning of the disease, the patient spreads the virus through the respiratory tract, and over time from the onset of the disease, the virus spreads more through the anal route $(25,26)$. Therefore, infected patients can potentially spread the virus through the respiratory tract, body fluid, and oral-fecal routes.

\section{Serological Test}

As mentioned, nasal and throat specimens are tested to identify people infected with COVID-19 using the RTPCR method. In some cases, the RT-PCR method cannot detect the infection (false-negative result); therefore, additional tests can be used to detect COVID-19 infection in people with a negative RT-PCR result. These include evaluating clinical signs, imaging tests, and serological tests (2).

Numerous researchers have developed serological methods for measuring antibody titers in patients' serum. In a study, Zhou et al used SARSr-CoV Rp3 NP as antigen to develop an anti-SARS-CoV IgG and IgM ELISA kit (26). 
In another study, Wang et al used a kind of nucleocapsid protein (NP) to developed IgM and IgG detection kit (27). These serological methods showed that the viral antibody titer increased over time from the onset of the disease (26).

For example, Zhang et al showed that the antibodies titer (IgM and IgG titer) was very low or even unmeasurable on the first day. In addition, an elevation of viral antibodies titer was seen in all patients on the fifth day. They suggested that both viral IgM and IgG serological test and the molecular test are needed to identify patients and carriers (25).

In general, the sensitivity of antibody tests in the first week after the onset of symptoms is very low and cannot play a major role in diagnosing COVID-19. It can also play a complementary role in other tests in people who show disease symptoms, though RT-PCR test is negative or not performed. If antibody tests are used on day 15 or later after the onset of symptoms, it can play an important role in diagnosing a previous SARS-CoV infection. However, the duration of antibody enhancement is currently unknown, and we found very little data for more than 35 days from the onset of symptoms (28).

\section{Laboratory Profiles}

Finding of complete blood counting (CBC) on admission demonstrated that $16-25 \%$ of patients had leucopenia (white blood cell $<4 \times 10^{9} / \mathrm{L}$ ); and over half of the patients (53.33\%-63\%) showed lymphopenia (lymphocyte count below $\left.1.0 \times 10^{9} / \mathrm{L}\right)(3,6,20)$. CD4+ lymphocyte count decreased significantly. Moreover, in some patients, the CD4+ lymphocyte /CD8+ lymphocyte ratio was lower than normal (19). Changes in hemoglobin and platelet counts have also been reported, which increased in some patients and were above the normal range, and reduced in some other patients (19). Coagulation impairments were observed in many patients, so that $58.67 \%$ of them had increased activated partial thromboplastin time and $40 \%$ had prolonged prothrombin time (19). Severe cases showed high D-dimer levels and prothrombin time (3, $7,19)$. Furthermore, $20-43 \%$ of patients developed liver dysfunctions, and the levels of alanine aminotransferase and aspartate aminotransferase were increased in their serum $(7,19,20)$. These elevations were much higher in ICU patients $(62 \%)$ than non-ICU patients $(25 \%)(3,19)$.

Some patients had an elevated rate of creatine kinase, hypersensitive troponin I (hs-cTnI), and lactate dehydrogenase $(\mathrm{LDH})$. The procalcitonin level was normal in most of them $(3,4,19,20)$. Moreover, the elevation of the erythrocyte sedimentation rate and C-reactive protein (CRP) was reported $(4,6,7,19)$.

In the study of Zhao et al, patients with elevated interleukin 6 (IL-6) showed significant decreases in the LYM\%, CD4+, and CD8+ T cell count (19). In addition, $7-20 \%$ of patients developed renal complications associated with elevated serum creatinine and blood urea nitrogen levels. These findings suggested that the internal organs could also be potential targets of COVID-19 (19, 20).

ICU-admitted patients had higher levels of white blood cells, especially neutrophils, as well as higher levels of D-dimer, creatine kinase, and creatine compared to nonICU patients $(18,29)$.

\section{Radiology finding}

Due to the large number of patients referring to healthcare systems in countries involved in the recent pandemic of COVID-19, the use of non-invasive diagnostic tools, fast, available, and with acceptable safety along with RTPCR is very important for better and faster management of patients. One of these diagnostic tools is the use of non-enhancing chest CT-scans (12). Various studies have shown that chest scans have higher sensitivity (86\%-98\%) for diagnosis of COVID-19 compared with initial RTPCR (18, 28-33).

A study conducted by $\mathrm{Ai}$ et al identified that $75 \%$ of patients, who had a negative RT-PCR results, had symptoms of COVID-19 on the chest CT scan (12). Moreover, $60-93 \%$ of cases had COVID-19 symptoms on the chest CT scan prior to or paralleled to the initial positive RT-PCR results (12). Several studies also showed that almost all patients with COVID-19 had characteristic symptoms on the chest CT scan $(5,12,34)$.

In a study conducted by Huang et al on 2019-nCoV infected patients, all patients had pneumonia with chest CT scan abnormalities (27). The hallmarks of COVID-19 infection on imaging were bilateral and peripheral ground-glass and consolidative pulmonary opacities (6, $18,20,27,35)$. For instance, several prospective analyses revealed that $71 \%-98 \%$ of the patients had bilateral involvements $(3,8,12,20), 46 \%-85 \%$ had ground-glass opacities, and $19 \%-50 \%$ had consolidations $(8,9,12,20)$. Moreover, opacities with a rounded morphology (33\%), reticulation (14\%), peripheral location of the opacities (33\%), consolidation with ground-glass opacities (29\%), and crazy-paving pattern (19\%) had been reported (8). In addition, it was found that as the disease progressed, the range of CT findings increased $(9,35)$.

The main features of chest CT scans of ICU patients on admission were subsegmental consolidation and bilateral multiple lobular areas. In contrast, non-ICU patients showed bilateral ground-glass opacity and subsegmental areas of consolidation. Subsequent chest CT scans showed bilateral ground-glass opacity, and the consolidation was resolved (3).

\section{Cytokine}

Measurement of cytokines in the acute phase of COVID-19 disease showed that plasma concentrations of TNFa, PDGF, MIP1B, MIP1A, MCP1, IP10, GCSF, GMCSF, IFN $\gamma$, IL10, IL9, IL8, IL7, IL1RA, IL1B, and VEGF were evaluated in these patients. Moreover, plasma 
Table 1. Clinical Symtoms, Laboratory and Radiological Findings of Patients Infected With COVID-19 (2, 5, 6, 8, 18, 19, 22, 25, 27, 28, 30-33)

\begin{tabular}{|c|c|c|}
\hline \multirow{2}{*}{$\begin{array}{l}\text { Disease Assessment } \\
\text { Methods }\end{array}$} & \multicolumn{2}{|l|}{ Findings } \\
\hline & Suggestive Findings & Inconsistent Findings \\
\hline Radiological findings & $\begin{array}{l}\text { Ground glass opacities (Bilateral, Multifocal, Unilateral, } \\
\text { Uniform) } \\
\text { - } \\
\text { Peripheral distribution or less likely peribronchovascular } \\
\text { distribution } \\
\text { - } \quad \text { Multifocal/bilateral consolidation (predominantly in late stage) } \\
\text { - Patterns of organizing pneumonia, reverse halo, linear opacities } \\
\text { and crazy-paving (might show in late stage) }\end{array}$ & $\begin{array}{ll}- & \text { Tree-in-bud opacities } \\
\text { - } & \text { Centrilobular nodules } \\
\text { - } & \text { Predominantly peribronchovascular distribution } \\
\text { - } & \text { Predominantly reticular opacities } \\
\text { - } & \text { Cavity } \\
\text { - } & \text { Lymphadenopathy } \\
\text { - } & \text { Pleural effusion }\end{array}$ \\
\hline Molecular findings & RT-PCR positive result, viral gene sequencing & \\
\hline Hematological findings & leukopenia, lymphopenia & Reduced CD4+/CD8+ ratio, coagulation disfunction \\
\hline Biochemical findings & & $\begin{array}{l}\text { Increased ALT, AST, hs-cTnl, creatine kinase, LDH, CRP, } \\
\text { ESR }\end{array}$ \\
\hline Serological findings & Elevation of viral $\lg M$ and $\lg G$ antibody some days after disease onset & \\
\hline Cytokine findings & $\begin{array}{l}\text { Elevation of TNF } \alpha \text {, PDGF, MIP1B, MIP1A, MCP1, IP10, basic FGF, } \\
\text { IFN }{ }_{\gamma} \text { GMCSF, GCSF, IL10, IL9, IL8, IL7, IL1RA, IL1B, and VEGF }\end{array}$ & $\begin{array}{l}\text { Elevation of TNF } \alpha, \text { MIP1A, MCP1, GCSF, IP10 IL7, IL10, } \\
\text { and IL2 }\end{array}$ \\
\hline clinical findings & fever, dry cough, dyspnea, chest tightness, anosmia, ageusia & $\begin{array}{l}\text { Sputum production, fatigue, myalgia, headache, } \\
\text { haemoptysis, diarrhoea,poor appetite, rash, sore throat, } \\
\text { shortness of breath, face pain, nasal obstruction, throat } \\
\text { congestion, tonsil swelling, enlargement of lymph nodes, } \\
\text { vomiting, consciousness disorder, paresthesia, stomach } \\
\text { ache }\end{array}$ \\
\hline
\end{tabular}

concentrations of TNFa, MIP1A, MCP1, IP10, GCSF, IL7, IL10, and IL2 were higher in ICU patients than non-ICU cases $(3,27)$.

Patients infected with 2019-nCoV also had high amounts of IL1B, IFN $\gamma$, IP10, and MCP1. Additionally, ICU-admitted patients had higher concentrations of GCSF, IP10, MCP1, MIP1A, and TNFa, suggesting that the cytokine storm was associated with disease severity (3).

In some patients with elevated IL-6, LYM\%, CD4+, and CD8+, T cell counts were significantly decreased, and NEU\%, CRP, and LDH levels increased significantly. Elevated IL-6 may be an important factor leading to T lymphocyte damage and cellular immune deficiency. IL- 6 could also be used as an indicator to evaluate infection severity (19).

Clinical symptoms, laboratory, and radiological findings of patients infected with COVID-19 are summarized in Table 1.

\section{Conclusion}

The reviewed studies showed that COVID-19 has a wide range of symptoms that occur in different people with different kinds of severity. The use of only one diagnostic method lacks sensitivity and accuracy and cannot diagnose COVID-19 with certainty, especially in the early stages of the disease. The main symptoms of the disease include respiratory symptoms, fever, cough, and dyspnea; moreover, there is a wide range of biochemical, hematological, and radiological changes in the patients. Finally, the signs (or symptoms) and other variables in the early stage or the mild stage of the disease appear in a highly heterogeneous and non-specific manner.

\section{Conflict of Interest Disclosure}

None to declare.

\section{Acknowledgement}

This research was supported by Ilam University of Medical Sciences, Ilam, Iran.

\section{Ethical Statement}

Not applicable.

\section{Authors' Contributions}

JF, IA, PM, HF, EZ, LK, ZF, and AF: Substantial contributions to the conception and design of the work; final approval of the version to be published; agreement to be accountable for all aspects of the work in ensuring that questions related to the accuracy or integrity of any part of the work are appropriately investigated and resolved. JF, ZF, HH, and HF: Analysis, interpretation of data for the work, final approval of the version to be published. MGB, MRB, SBN, and HM: Agreement to be accountable for all aspects of the work in ensuring that questions related to the accuracy or integrity of any part of the work are appropriately investigated and resolved; final approval of the version to be published.

\section{Funding/Support}

This research was supported by Gerash University of Medical science, Gerash, Iran.

\section{Informed Consent}

Informed Consent Not applicable because it was based on searching in databases

\section{References}

1. Lai CC, Shih TP, Ko WC, Tang HJ, Hsueh PR. Severe acute respiratory syndrome coronavirus 2 (SARS-CoV-2) and 
coronavirus disease-2019 (COVID-19): the epidemic and the challenges. Int J Antimicrob Agents. 2020;55(3):105924. doi: 10.1016/j.ijantimicag.2020.105924.

2. World Health Organization (WHO). Laboratory Testing for Coronavirus Disease 2019 ('COVID-19)| in Suspected Human Cases: Interim Guidance, 2 March 2020. WHO; 2020.

3. Zhu N, Zhang D, Wang W, Li X, Yang B, Song J, et al. A novel coronavirus from patients with pneumonia in China, 2019. N Engl J Med. 2020;382(8):727-33. doi: 10.1056/ NEJMoa2001017.

4. Chan JF, Yuan S, Kok KH, To KK, Chu H, Yang J, et al. A familial cluster of pneumonia associated with the 2019 novel coronavirus indicating person-to-person transmission: a study of a family cluster. Lancet. 2020;395(10223):514-23. doi: 10.1016/s0140-6736(20)30154-9.

5. Lei J, Li J, Li X, Qi X. CT imaging of the 2019 novel coronavirus (2019-nCoV) pneumonia. Radiology. 2020;295(1):18. doi: 10.1148/radiol.2020200236.

6. Chen ZM, Fu JF, Shu Q, Chen YH, Hua CZ, Li FB, et al. Diagnosis and treatment recommendations for pediatric respiratory infection caused by the 2019 novel coronavirus. World J Pediatr. 2020;16(3):240-6. doi: 10.1007/s12519-02000345-5.

7. Shen K, Yang Y, Wang T, Zhao D, Jiang Y, Jin R, et al. Diagnosis, treatment, and prevention of 2019 novel coronavirus infection in children: experts' consensus statement. World J Pediatr. 2020;16(3):223-31. doi: 10.1007/s12519-020-00343-7.

8. Chung M, Bernheim A, Mei X, Zhang N, Huang M, Zeng $X$, et al. CT imaging features of 2019 novel coronavirus (2019-nCoV). Radiology. 2020;295(1):202-7. doi: 10.1148/ radiol.2020200230.

9. Pan Y, Guan H, Zhou S, Wang Y, Li Q, Zhu T, et al. Initial CT findings and temporal changes in patients with the novel coronavirus pneumonia (2019-nCoV): a study of 63 patients in Wuhan, China. Eur Radiol. 2020;30(6):3306-9. doi: 10.1007/ s00330-020-06731-x.

10. Chang L, Yan Y, Wang L. Coronavirus disease 2019: coronaviruses and blood safety. Transfus Med Rev. 2020;34(2):75-80. doi: 10.1016/j.tmrv.2020.02.003.

11. Mahase E. China coronavirus: WHO declares international emergency as death toll exceeds 200. BMJ. 2020;368:m408. doi: 10.1136/bmj.m408.

12. Ai T, Yang Z, Hou H, Zhan C, Chen C, Lv W, et al. Correlation of chest CT and RT-PCR testing for coronavirus disease 2019 (COVID-19) in China: a report of 1014 cases. Radiology. 2020;296(2):E32-E40. doi: 10.1148/radiol.2020200642.

13. Poortahmasebi V, Zandi M, Soltani S, Jazayeri SM. Clinical performance of RT-PCR and chest CT scan for COVID-19 diagnosis; a systematic review. Adv J Emerg Med. 2020;4(2S):E57. doi: 10.22114/ajem.v4i2s.459.

14. Fani M, Zandi M, Soltani S, Abbasi S. Future developments in biosensors for field-ready SARS-CoV-2 virus diagnostics. Biotechnol Appl Biochem. 2020. doi: 10.1002/bab.2033.

15. Gane SB, Kelly C, Hopkins C. Isolated sudden onset anosmia in COVID-19 infection. A novel syndrome? Rhinology. 2020;58(3):299-301. doi: 10.4193/Rhin20.114.

16. Gautier JF, Ravussin Y. A new symptom of COVID-19: loss of taste and smell. Obesity (Silver Spring). 2020;28(5):848. doi: 10.1002/oby.22809.

17. Lechien JR, Chiesa-Estomba CM, De Siati DR, Horoi M, Le Bon SD, Rodriguez A, et al. Olfactory and gustatory dysfunctions as a clinical presentation of mild-to-moderate forms of the coronavirus disease (COVID-19): a multicenter European study. Eur Arch Otorhinolaryngol. 2020;277(8):2251-61. doi: 10.1007/s00405-020-05965-1.

18. Wang D, Hu B, Hu C, Zhu F, Liu X, Zhang J, et al. Clinical characteristics of 138 hospitalized patients with 2019 novel coronavirus-infected pneumonia in Wuhan, China. JAMA. 2020;323(11):1061-9. doi: 10.1001/jama.2020.1585.

19. Zhao Z, Xie J, Yin M, Yang Y, He H, Jin T, et al. Clinical and laboratory profiles of 75 hospitalized patients with novel coronavirus disease 2019 in Hefei, China. medRxiv. 2020. doi: 10.1101/2020.03.01.20029785.

20. Chen N, Zhou M, Dong X, Qu J, Gong F, Han Y, et al. Epidemiological and clinical characteristics of 99 cases of 2019 novel coronavirus pneumonia in Wuhan, China: a descriptive study. Lancet. 2020;395(10223):507-13. doi: 10.1016/s0140-6736(20)30211-7.

21. Wang W, Xu Y, Gao R, et al. Detection of SARSCoV-2 in Different Types of Clinical Specimens. JAMA. 2020;323(18):1843-1844. doi:10.1001/jama.2020.3786.

22. Yang Y, Yang M, Shen C, Wang F, Yuan J, Li J, et al. Evaluating the accuracy of different respiratory specimens in thelaboratory diagnosis and monitoring the viral shedding of 2019-nCoV infections. medRxiv. 2020

23. Siordia JA Jr. Epidemiology and clinical features of COVID-19: a review of current literature. J Clin Virol. 2020;127:104357. doi: 10.1016/j.jcv.2020.104357.

24. Huang X, Wei F, Hu L, Wen L, Chen K. Epidemiology and clinical characteristics of COVID-19. Arch Iran Med. 2020;23(4):268-71. doi: 10.34172/aim.2020.09.

25. Zhang $W$, Du RH, Li B, Zheng XS, Yang XL, Hu B, et al. Molecular and serological investigation of 2019nCoV infected patients: implication of multiple shedding routes. Emerg Microbes Infect. 2020;9(1):386-9. doi: 10.1080/22221751.2020.1729071.

26. Zhou P, Yang XL, Wang XG, Hu B, Zhang L, Zhang W, et al. Discovery of a novel coronavirus associated with the recent pneumonia outbreak in humans and its potential bat origin. bioRxiv. 2020. doi: 10.1101/2020.01.22.914952.

27. Wang N, Li SY, Yang XL, Huang HM, Zhang YJ, Guo H, et al. Serological evidence of bat SARS-related coronavirus infection in humans, China. Virol Sin. 2018;33(1):104-7. doi: 10.1007/s12250-018-0012-7.

28. Deeks JJ, Dinnes J, Takwoingi Y, Davenport C, Spijker R, TaylorPhillips S, et al. Antibody tests for identification of current and past infection with SARS-CoV-2. Cochrane Database Syst Rev. 2020;6(6):CD013652. doi: 10.1002/14651858.cd013652.

29. Huang C, Wang Y, Li X, Ren L, Zhao J, Hu Y, et al. Clinical features of patients infected with 2019 novel coronavirus in Wuhan, China. Lancet. 2020;395(10223):497-506. doi: 10.1016/s0140-6736(20)30183-5.

30. Xie X, Zhong Z, Zhao W, Zheng C, Wang F, Liu J. Chest CT for typical 2019-nCoV pneumonia: relationship to negative RTPCR testing. Radiology. 2020;296(2):E41-E5. doi: 10.1148/ radiol.2020200343.

31. Ai T, Yang Z, Hou H, Zhan C, Chen C, Lv W, et al. Correlation of chest CT and RT-PCR testing in coronavirus disease 2019 (COVID-19) in China: a report of 1014 cases. Radiology. 2020;296(2):E32-E40. doi: 10.1148/radiol.2020200642.

32. Guan WJ, Ni ZY, HuY, Liang WH, Ou CQ, He JX, et al. Clinical characteristics of coronavirus disease 2019 in China. N Engl J Med. 2020;382(18):1708-20. doi: 10.1056/NEJMoa2002032.

33. Udugama B, Kadhiresan P, Kozlowski HN, Malekjahani A, Osborne M, Li VYC, et al. Diagnosing COVID-19: the disease and tools for detection. ACS Nano. 2020;14(4):3822-35. doi: 
10.1021/acsnano.0c02624.

34. Huang P, Liu T, Huang L, Liu H, Lei M, Xu W, et al. Use of chest $C T$ in combination with negative RT-PCR assay for the 2019 novel coronavirus but high clinical suspicion. Radiology. 2020;295(1):22-3. doi: 10.1148/radiol.2020200330.
35. Bernheim A, Mei X, Huang M, Yang Y, Fayad ZA, Zhang $\mathrm{N}$, et al. Chest CT findings in coronavirus disease-19 (COVID-19): relationship to duration of infection. Radiology. 2020;295(3):200463. doi: 10.1148/radiol.2020200463. 